\title{
Energy degrader technique for light-charged particle spectroscopy at LOHENGRIN
}

\author{
A. Oberstedt ${ }^{1, \mathrm{a}}$, S. Oberstedt ${ }^{2, \mathrm{~b}}$, and D. Rochman ${ }^{2, \mathrm{c}}$ \\ ${ }^{1}$ Institutionen för Naturvetenskap, Örebro Universitet, 70182 Örebro, Sweden \\ ${ }^{2}$ Institut Laue-Langevin, 6 rue Horowitz, 38042 Grenoble, France
}

\begin{abstract}
The recoil mass-separator LOHENGRIN at Institute Laue-Langevin was originally designed for the spectrometry of binary fission fragments. Nevertheless, it was also used in the past for measuring light-charged particles from ternary fission. However, due to the electric field settings the energy distribution of the lightest particles was not completely accessible, which made the determination of mean kinetic energies, widths and, hence, emission yields difficult. In this paper we present an energy degrader technique that allows for the measurement of the entire energy spectrum of even the lightest ternary particles with LOHENGRIN.
\end{abstract}

\section{Introduction}

The unambiguous identification of light charged particles (LCP), e.g., produced in nuclear fission, is a necessary prerequisite for the full understanding of the nature of their formation. Solid state detectors, assembled as $\Delta \mathrm{E}-\mathrm{E}$ telescope, may provide a separation of atomic numbers, while time-offlight measurements may give information of the particles' mass. A severe disadvantage, however, is the fact that particles with low kinetic energies are stopped in the $\Delta \mathrm{E}$-detector and, thus, remain unidentified. Moreover, since the solid state detectors have to be protected from the intense flux of binary fission fragments, thin absorber foils are usually installed in front of the detectors. This makes it often difficult to assess the entire energy distribution (see fig. 1.) As an alternative, a mass-separator in combination with an ionization chamber with split anode, the latter serving as $\Delta \mathrm{E}-\mathrm{E}$ telescope and being available at Institute Laue-Langevin (ILL), may be employed. In a recent paper [1] we presented a technique that allows to adapt the fission fragment mass spectrometer LOHENGRIN to the detection of ternary particles over the full kinetic energy range.

\section{Background}

The recoil mass-separator LOHENGRIN was originally designed for binary fission fragments produced at the high flux reactor of Institute Laue-Langevin in Grenoble [3]. The fission products are separated by electric and magnetic fields according to the equations

$$
m / q=\left(\rho_{m a g}^{2} / \rho_{e l}\right) B^{2} d / U
$$

and

$$
E / q=\left(\rho_{e l} / 2\right) d / U
$$

\footnotetext{
${ }^{a}$ Present author, e-mail: andreas . oberstedt@nat.oru.se

${ }^{b}$ Present address: EC-JRC Institute for Reference Materials and Measurements (IRMM), 2440 Geel, Belgium

${ }^{c}$ Present address: Brookhaven National Laboratory, National Nuclear Data Center, Upton, NY 11973-5000, USA
}

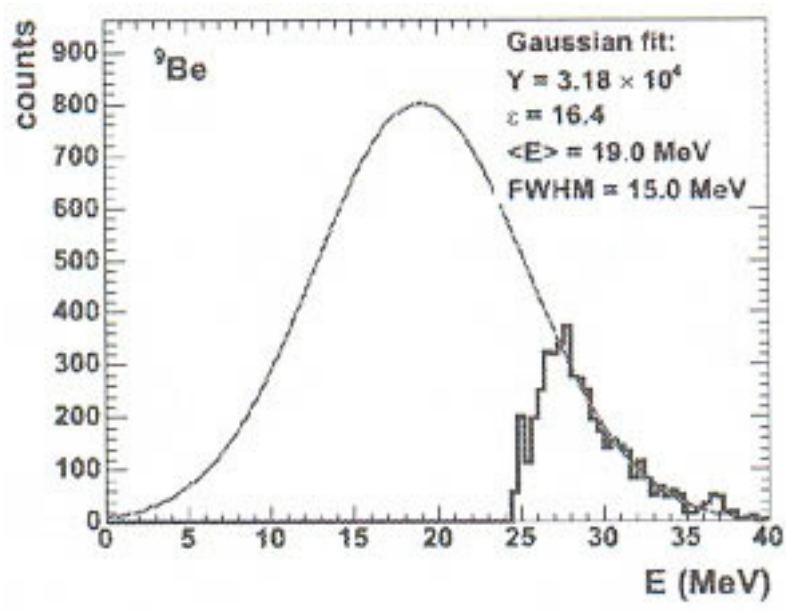

Fig. 1. Energy spectrum of ternary ${ }^{9} \mathrm{Be}$ from the spontaneous fission of ${ }^{252} \mathrm{Cf}$, taken with $\Delta \mathrm{E}-\mathrm{E}_{\text {rest }}$ telescopes as reported in ref. [2].

The particles' mass, ionic charge and kinetic energy are denoted by $\mathrm{m}, \mathrm{q}$ and $\mathrm{E}$, respectively, while $\mathrm{B}$ is the magnetic flux density and $\mathrm{U} / \mathrm{d}$ the electric field in the capacitor. The deflection radii of the magnet and the capacitor are represented by $\rho_{\text {mag }}$ and $\rho_{e l}$, respectively.

The maximum electric field settings of the LOHENGRIN spectrometer correspond to a maximum measurable particle kinetic energy

$$
E_{L}=5.5 \mathrm{MeV} \times q,
$$

where $E_{L}$ stands for the kinetic energy with which the particle actually passes the mass spectrometer. In the past, also light charged particles from ternary fission have been detected with LOHENGRIN and mean kinetic energies and widths of their energy distributions as well as emission yields were extracted (e.g., [4-7]).

However, in some cases the complete energy distribution was not measurable because of the kinematic limits given by equation (3). For instance, the energy spectrum of ${ }^{9} \mathrm{Be}$, emitted in the reaction ${ }^{235} \mathrm{U}\left(\mathrm{n}_{\mathrm{th}}, \mathrm{f}\right)$ ranges up to $\mathrm{E}>35 \mathrm{MeV}$, whereas the maximum LOHENGRIN energy for ${ }^{9} \mathrm{Be}^{3+}$ ions is about 


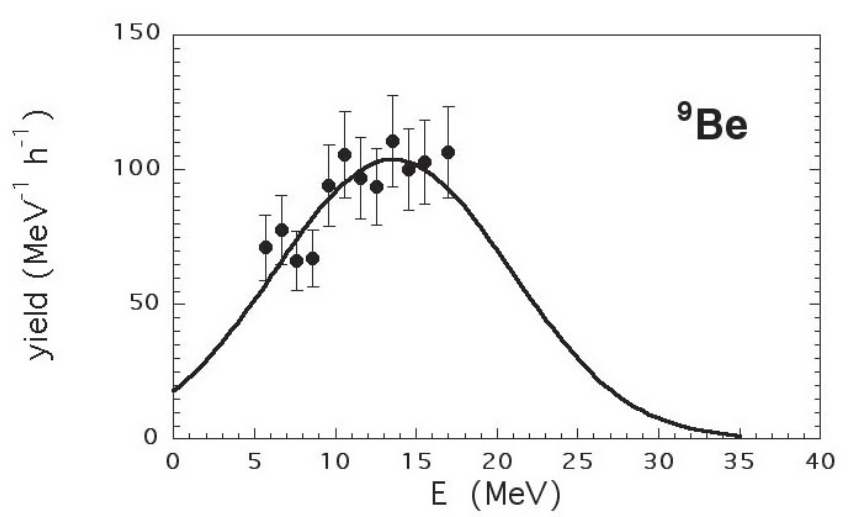

Fig. 2. Energy spectrum of ternary ${ }^{9} \mathrm{Be}$ from the reaction ${ }^{235} \mathrm{U}\left(\mathrm{n}_{\mathrm{th}}, \mathrm{f}\right)$, measured at LOHENGRIN (this work).

$22 \mathrm{MeV}$ (cf. fig. 2). Consequently, a description of the energy distributions of the lightest LCPs, usually parameterized by Gaussians, is difficult, if not both tails of the distribution are sufficiently well known.

In order to make practically the entire energy spectra of the lightest ternary particles accessible with the LOHENGRIN mass-separator, we suggest to apply an energy degrader to reduce the initially high kinetic energies to those in compliance with equation (3). Below we discuss the influence of the energy degrader on the measured particle spectra and present results from a Monte Carlo simulation that shows how the original energy distributions are reconstructed. Finally, we apply this procedure to experimental data.

\section{Monte Carlo simulation}

In order to slow down high energetic light ions prior to entering the mass spectrometer, we suggest to put a degrader foil (e.g., nickel) of appropriate thickness into the particle beam between target and spectrometer entrance. From the choice of energy, corresponding to the spectrometer's field settings for a given ionic charge state and the properties of the degrader material, the kinetic energy, with hich the particles were emitted in the fission process, can be calculated according to their energy loss in the degrader foil. The use of an energy degrader affects the particle beam in two ways: for one, the particles will have a reduced kinetic energy before they enter the spectrometer; secondly, due to the constant energy acceptance of the mass separator $\delta \mathrm{E} / \mathrm{E}$, less particles pass the separator because of their lower kinetic energy. As a consequence, we expect to observe a reduced yield at a reduced energy in the spectrum.

Figure 3 shows the result of a Monte Carlo simulation for a typical LCP energy distribution. We have chosen a Gaussian with the following characteristics: an average energy of $15 \mathrm{MeV}$, a width of about $11 \mathrm{MeV}$ (FWHM), and 1000 particles. The full drawn line shows the energy distribution and the full black circles the randomly obtained yield, i.e., number of particles per energy unit, for selected energies. For the energy loss we assume that events with $\mathrm{E}<12 \mathrm{MeV}$ are stopped, while those with $\mathrm{E}=30 \mathrm{MeV}$ leave the degrader

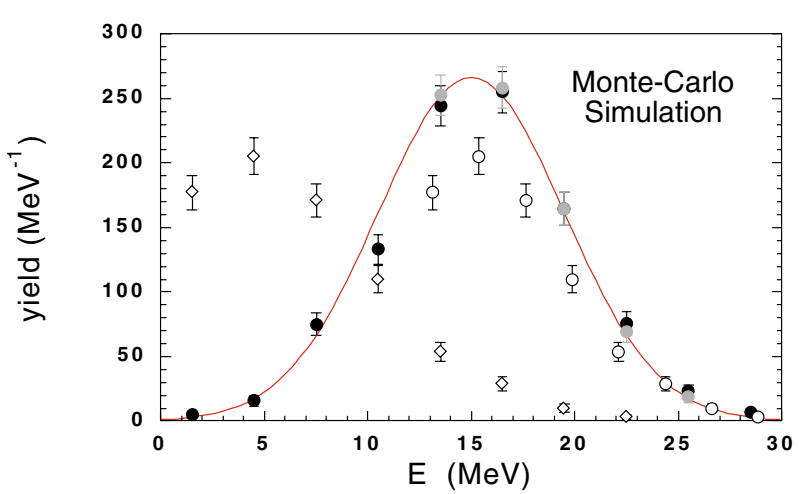

Fig. 3. Result of a Monte-Carlo simulation of particles passing an energy degrader. The original energy distribution is shown as full drawn line and full black circles. The open diamonds denote the corresponding yield distribution obtained by calculating the residual energies for each event. The open circles show the same yields, but now plotted as a function of incident energy, whereas the recreated original distribution is shown as full grey circles.

foil with $\mathrm{E}_{\text {res }}=24 \mathrm{MeV}$. According to results of energy loss calculations performed with the computer code SRIM [8], the residual energy for incident energies between 12 and $30 \mathrm{MeV}$ may be obtained by linear extrapolation. If one calculates now the residual energies behind the degrader for each event and determines their number per $\mathrm{MeV}$, one obtains the distribution shown as open diamonds in figure 3. Moving these points to their correct, i.e., initial, energies results in the open circles. Obviously, the yields are underestimated, which is due to the fact, that the spectrometer lets through fewer particles with reduced energy than it would be the case without energy degrader. This effect can be corrected for by multiplying these yields with the energy ratio $\mathrm{E} / \mathrm{E}_{\text {res }}$ at a given energy E. However, the transformation from $\mathrm{E}_{\text {res }}$ to $\mathrm{E}$ in the plot leads to an effective binsize that is less than $1 \mathrm{MeV}$. Hence, the yields have to be multiplied as well by the derivative $\left(\mathrm{dE} / \mathrm{dE}_{\text {res }}\right)$, which is less than 1. Applying both corrections and binning around the same energies as indicated by the black dots, leads finally to the original energy distribution, now shown as grey dots. We observe a very nice agreement, of course only for events with energies large enough to pass the degrader foil. In the following section we apply the method described above to the measured energy spectrum of ${ }^{9} \mathrm{Be}$ produced in the reaction ${ }^{235} \mathrm{U}\left(\mathrm{n}_{\mathrm{th}}, \mathrm{f}\right)$, as shown in section 2.

\section{Experimental verification}

High resolution measurements of ternary particles produced in the thermal neutron-induced fission of ${ }^{236} \mathrm{U}^{*}$ were performed at the high-flux reactor at ILL $\left(\Phi_{n}=5.4 \times 10^{14} \mathrm{~cm}^{-2} \mathrm{~s}^{-1}\right)$. The target consisted of ${ }^{235} \mathrm{UO}_{2}$ of $500 \mu \mathrm{g} / \mathrm{cm}^{2}$ thickness and a size of $7.0 \times 0.5 \mathrm{~cm}^{2}$. In order to extend the kinetic energy of the LCPs measurable with the mass-separator LOHENGRIN, we introduced a $10 \mu \mathrm{m}$ thick Ni-foil as energy degrader. The details about this experiment as well as the presentation of all results will be found elsewhere [9], here our intention is 


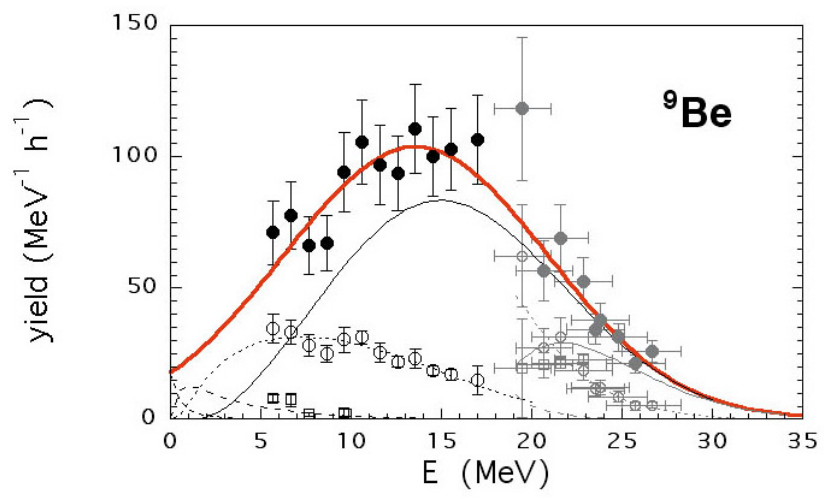

Fig. 4. Experimental energy distribution for ${ }^{9} \mathrm{Be}$, measured with and without energy degrader (grey and black symbols, respectively). The open squares correspond to ${ }^{9} \mathrm{Be}^{2+}$, the open circles to ${ }^{9} \mathrm{Be}^{3+}$, and the full circles to the sum of all charge states. The thick, full drawn line corresponds to a Gaussian fitted to all experimental data, while the full drawn, dotted, long and short dashed lines indicate the calculated $4+, 3+, 2+$ and $1+$ contributions, respectively.

to demonstrate the value of the degrader technique. Figure 4 shows the measured energy distribution of ${ }^{9} \mathrm{Be}$. The experimental data were corrected for target burn-up and energy loss according to the procedures described, e.g., in ref. [4]. The Be-ions were measured for charge states $\mathrm{q}=3^{+}$and $2^{+}$, depicted in figure 4 as open circles and open squares, respectively. The full circles show the total yield, integrated over all ionic charge states. The probability for the different charge states as a function of kinetic energy was calculated according to a description given in ref. [10], reproducing the experimental data. Since the contributions of $0^{+}$and $1^{+}$ are negligible at the measured energies, the total yields were determined by adding those for $2^{+}$and $3^{+}$and correcting the missing $4^{+}$contributions with the calculated ionic charge state distribution. All data obtained without energy degrader are shown in black, the ones taken with energy degrader are depicted in grey. The thick, full drawn line corresponds to a Gaussian fitted to all experimentally obtained yields. The full drawn, dotted and dashed lines indicate the calculated contributions of ${ }^{9} \mathrm{Be}^{4+},{ }^{9} \mathrm{Be}^{3+}$ and ${ }^{9} \mathrm{Be}^{2+}$, respectively, whereas the ${ }^{9} \mathrm{Be}^{1+}$ contribution is hardly visible (short dashed lines). The error bars contain statistical errors and, in case the energy degrader was used, systematic uncertainties from the different corrections applied. In the latter case an uncertainty in energy was estimated from the linear fit to the $\mathrm{E}_{-} \mathrm{E}_{\text {res }}$ dependence calculated with SRIM [8]. In comparison, the energy uncertainty due to energy straggling, which is of the order of less than $1 \%$ of the particle's incident energy in the Gaussian limit, may be neglected. Again we refer to the detailed description in ref. [9].

The degrader data shown as open symbols are already shifted to the correct energies and correspond, thus, to the open circles in figure 3 . The yields were then corrected as described in the previous chapter. The excellent agreement between data taken with and without energy degrader is obvious. Furthermore, even the symmetric shape of the Gaussian for both the low and the high-energy tail of the spectrum makes us confident in the presented energy degrader technique.

\section{Summary and conclusion}

In this paper we have presented an experimental technique to increase the kinematical limits of mass-separators in general by using an energy degrader that reduces the kinetic energies of an entering particle beam. By means of Monte Carlo simulations the effect of the energy degrader on a measured energy spectrum was demonstrated and explained. We have also shown how the original energy distribution easily is constructed from the observed, distorted one. The only information that is required is the dependence between initial and residual energy, which may be determined by energy loss calculations.

Here we have applied this technique on the high resolution measurement of ternary ${ }^{9} \mathrm{Be}$ produced in the reaction ${ }^{235} \mathrm{U}\left(\mathrm{n}_{\mathrm{th}}, \mathrm{f}\right)$ and found an excellent agreement between data taken with and without energy degrader. In ref. [1] the application of the energy degrader technique was demonstrated on ${ }^{7} \mathrm{Li}$ from the same reaction. There we have also shown that the influence of lateral straggling on the detected particle yields is negligible. Using a nickel foil of $10 \mu \mathrm{m}$ thickness the energy range of the spectrometer for ${ }^{9} \mathrm{Be}^{3+}$ was extended to about $27 \mathrm{MeV}$, exceeding the kinematical limit given in equation (3) by about $60 \%$. Rephrasing equation (3) for effective LOHENGRIN energies $E_{L, \text { eff }}$, leads to

$$
E_{L, e f f}=9.0 \mathrm{MeV} \times q,
$$

which of course is depending on the LCP, its ionic charge state and the choice of energy degrader material. The connection between $E_{L, e f f}$ and $E_{L}$ is given by

$$
E_{L, e f f}=E_{L}+\Delta E\left(E_{L}\right)
$$

where $\Delta \mathrm{E}\left(E_{L}\right)$ denotes the energy loss in the degrader. We would like to conclude with expressing our confidence in that this technique is a valuable tool for the high precision spectroscopy of especially the lightest charged particles, that is $\mathrm{He}$ - and Li-isotopes, with mass spectrometers like LOHENGRIN. Further evidence for that will be given in ref. [9]. For the future, in particular, the emission of ternary ${ }^{2,3} \mathrm{H}$ and ${ }^{4} \mathrm{He}$ needs a revision. Previous results for the most probable energies and widths (FWHM) for these nuclides, $\bar{E} \approx 8 \mathrm{MeV}$ and $\mathrm{FWHM} \approx 7 \mathrm{MeV}$ for the $\mathrm{H}$-isotopes and $\bar{E} \approx 16 \mathrm{MeV}$ and $\mathrm{FWHM} \approx 10 \mathrm{MeV}$ for ${ }^{4} \mathrm{He}$, obtained at LOHENGRIN [11], were far beyond the kinematical limits. The energy degrader technique offers the possibility for new, precise measurements of the essentially complete energy distributions.

\section{References}

1. A. Oberstedt, S. Oberstedt, Nucl. Instrum. Meth. A 570, 51 (2007).

2. Yu.N. Kopatch, V. Tishchenko, M. Speransky, M. Mutterer, F. Gönnenwein, P. Jesinger, A.M. Gagarski, J. von Kalben, I. Kojouharov, E. Lubkiewics, Z. Mezentseva, V. Nezvishevsky, G.A. Petrov, H. Schaffner, H. Scharma, W.H. Trzaska, H.-J. Wollersheim, in Nucl. Fission and Fission Spectroscopy: Third Int. Workshop, edited by H. Goutte, H. Faust, G. Fioni, D. Goutte, AIP Conf. Proc. 798 (2005), p. 115. 
3. E. Moll, H. Schrader, G. Siegert, H. Hammers, M. Asghar, J.P. Bocquet, P. Armbruster, H. Ewald, H. Wollnik, Kerntechnik 8, 374 (1977).

4. S. Oberstedt, A. Oberstedt, D. Rochman, F. Gönnenwein, I. Tsekhanovich, J. Becker, A. Sartz, H. Bax, F.-J. Hambsch, S. Raman, Nucl. Phys. A 761, 173 (2005).

5. I. Tsekhanovich, Z. Büyükmumcu, M. Davi, H.O. Denschlag, F. Gönnenwein, S.F. Boulyga, Phys. Rev. C 67, 034610 (2003).

6. U. Köster, H. Faust, G. Fioni, T. Friedrichs, M. Groß, S. Oberstedt, Nucl. Phys. A 652, 371 1999).
7. M. Wöstheinrich, R. Pfister, F. Gönnenwein, H.O. Denschlag, H. Faust, S. Oberstedt, in Nucl. Fission and Fission Spectroscopy: Second Int. Workshop, edited by G. Fioni, H. Faust, F.-J. Hambsch, S. Oberstedt, AIP Conf. Proc. 447, 1998, p. 330.

8. Computer code SRIM 2000.39, available from J.F. Ziegler, IBM Research, Yorktown, NY-10598, USA and J.P. Biersack, HahnMeitner Institut, Berlin-39, Germany.

9. A. Oberstedt et al. (to be published).

10. Y. Baudinet-Robinet, Nucl. Instrum. Meth. 190, 197 (1981).

11. W. Baum, Dissertation D17, Technische Universität Darmstadt, 1992 (and private communication). 